\title{
An Absorption Tube for the Investigation of Gases in the Photographic Infrared
}

Richard M. Badger, Lyman G. Bonner and Paul C. Cross, Gates Chemical Laboratory, California Institute of Technology

(Received August 3, 1935)

An absorption cell of novel design is described, which combines the advantages of long path and relatively small volume.

$I^{\mathrm{N}}$ $\mathrm{N}$ the course of investigating the absorption of some polyatomic gases in the photographic infrared, in this laboratory, it was found that many interesting substances have a rather weak absorption which necessitates the presence of a very considerable amount of the material to be studied in the light path, to obtain absorption bands strong enough to photograph. This condition cannot in general be attained by using a high pressure of gas in a short absorption cell, since we have found in most cases no advantage in using pressures above one or two atmospheres due to the pressure broadening effect. For example, when the lines of an absorption band photographed at one atmosphere pressure are barely distinguishable on a spectrogram, because of weak absorption, an increase to three atmospheres in the same cell will in general result in little or no improvement as the increase in diffuseness of the lines counterbalances the greater absorption.

Consequently, in order to carry out a program of investigation which we had outlined, it appeared that an absorption cell of considerable length was necessary. An additional and conflicting requirement on the cell was that it have as small a volume as possible, since many of the substances to be investigated are difficult to obtain in large quantities.

It appeared to us that these two requirements were most easily satisfied by departing from the usual construction and using a tube of relatively small diameter with highly polished interior walls which would serve in place of the usual optical system to confine the light in the desired path. We accordingly constructed a cell seventy feet in length from stainless steel tubing of approximately $7 / 8$ in. internal diameter, which had been provided by the manufacturer with a rather high polish on the interior wall. The tubing was obtained in five equal sections which were bolted together by means of flanges welded to the ends. A tongue or groove, respectively, in the opposing faces of a pair of flanges and a rubber ring gasket seated in the groove provide a satisfactory vacuum tight seal. The ends of the tube were closed with plates provided with plate glass windows seated in recesses and made tight either with sealing wax or rubber gaskets. Arrangements for evacuating the tube and for introducing various gases were provided.

Since it was proposed to study some substances which have relatively high boiling points, it was necessary to provide means of heating the tube. It was found that this was most conveniently done by passing a current through the tube itself. After the tube had been covered with ordinary steam pipe insulation it was found that a current of 86 amperes will maintain it a temperature of about $100^{\circ} \mathrm{C}$. A potential of about 14 volts is required to pass this current.

It was somewhat difficult to predict what the optical efficiency of the absorption cell would be, but some estimates were made which were a guide in choosing the diameter of tubing to be used. The aperture of the spectrograph used in the investigations is such that of the light emerging from the absorption cell and entering the spectrograph slit only that making an angle of less than about $40^{\prime}$ with the cell axis will be caught by the grating. Because of this small angle the useful light in passing through the tube will have been reflected from the walls a maximum of ten times, and the greater part of it several times fewer. Furthermore, the reflection at these small angles is very efficient in the infrared, even with a relatively poorly reflecting material. The optical constants of the tube material were not available but assuming that they are not greatly different from those of ordinary high carbon 
steel we estimated that our tube would be 75-80 percent as good as one made from a perfectly reflecting material.

This estimate was made on the assumption that the tube is a perfect cylinder, which it is not since it is slightly bowed in places and has minute ripples on the interior wall. However, we have recently made experiments in which a system of lenses was introduced into the cell which did not result in material improvement and showed that the arrangement worked nearly as well as could have been hoped.

\section{Method of Illumination}

The light source found most satisfactory is the ordinary double-filament automobile headlamp bulb. The two filaments are used simultaneously being connected in series, and are run at considerable overvoltage so that the life of the lamp is 24 hours or less. Not only is the cost of the lamps so low that a new one may be used for every exposure, if necessary, but they have proved as satisfactory as much more elaborate and expensive lamps. The light from the source is made nearly parallel as it enters the cell by means of the most powerful condensing lens practicable, which is fastened to the tube window. The position of the lamp is of course rather critical and micrometer screw adjustments are provided on its mounting.

For practical reasons the emergence end of the cell had to be about four feet from the spectrograph slit, necessitating the use of a lens between the two. We have had best results with a lens of about $70 \mathrm{~cm}$ focal length placed somewhat less than this distance from the slit.

\section{The SPectrograph}

The spectrograph used in conjunction with the absorption cell employs a $21-\mathrm{ft}$. concave grating with a $3 \times 6$-in. ruled surface, in an Eagle mounting. The grating was especially ruled for the purpose and in the photographic infrared throws most of the light in the first order on one side. With the arrangements as described and using Eastman infrared plates sensitized with ammonia the exposure times required are usually quite reasonable. Between the visible and $\lambda 10,300$, where the sensitivity of the Eastman $Q$ plates falls off rapidly, the exposure time ranges from fifteen minutes to four hours, provided the appropriate plates are used in each region. 